\title{
Chronic Active Colitis
}

National Cancer Institute

\section{Source}

National Cancer Institute. Chronic Active Colitis. NCI Thesaurus. Code C82954.

Chronic colitis with superimposed neutrophilic mucosal infiltrates. It is usually seen during exacerbations of inflammatory bowel disease. 